\title{
A ROBUST APPROACH IN NONLINEAR FUZZY CONTROL OF CHEMICAL PROCESS
}

\author{
Behnam Baloochy * \\ Department of Modeling and Process Control, Technology Development Research Division, Research Institute of \\ Petroleum Industry, Tehran, Iran
}

Received 21 November 2012; received in revised form 17 September 2013

\begin{abstract}
This paper presents a novel and robust fuzzy controller. The controller has conventional fuzzy inputs membership functions but has Bang-bang subsequent outputs membership functions. The controller on-line adaptation is accomplished by tuning and selforganizing the input membership functions. New rules and membership functions are created online with sliding mode control knowledge base rules. The system's closed loop stability is assured for each rule, which is stable in sense of Lyapunov. The suggested controller performance and analysis is confirmed by simulation of well know non-linear benchmark problems.
\end{abstract}

Keywords: Nonlinear Control; Fuzzy Logic; Chemical Process; Knowledge Base; Lyapunov Stability.

\section{INTRODUCTION}

Nonlinear control is the area of control engineering specifically involved with systems that are nonlinear, timevariant, or both. As the complexity of the control system tasks is increased, so does the difficulty of mathematical modeling of such nonlinear system. The presence of inevitable un-modeled nonlinearities and uncertain disturbances makes the conventional control techniques ineffective [1-2]. Fuzzy controller applications [3-6] are on rise due to their simplicity in design and imperviousness to the mathematical modeling and un-modeled dynamics. Simple fuzzy controllers have fixed parameter, structure and rules to take care of wellbounded control parameters. However, in adverse situations, where the plant parameters are subjected to unknown disturbance, adaptive schemes are used

*To whom all correspondence should be addressed. (e-mail: baloochyb@ripi.ir) which automatically alters the controller's parameters to absorb the disturbance without losing the control of the plant.

The classical adaptive control requires parametric estimates of plant states. Feedback or Jacobian linearization techniques are used for cancellation of plant nonlinear terms, which are difficult to apply in uncertain conditions $[1,7,8]$. On the other hand fuzzy adaptive control uses the universal approximation property $[9,10]$ of the fuzzy logic system (FLS). The good thing about the adaptive fuzzy controllers is that the adaptive laws are linguistic and heuristically - based on human instinct to control the system. This adaptive law fulfils the Lyaponov stability criteria under bounded conditions [11]. 
Adaptive approaches can be direct or indirect. Indirect adaptive Fuzzy controller uses Takagi-Sugeno fuzzy method [4, 12, $13,14,15]$ to model the non-linear plant The control signal to counter the changes in plant parameters is then achieved by adaptively adjusting the centers of consequent membership functions of the fuzzy basis, and hence the controller is synthesizes from the plant estimates. For direct scheme where the controller parameters are estimated directly, Park J.H et.al [16] uses feedback linearization techniques, S. Laboid and T.H. Guerra [17] and others $[11,14,18]$ uses state feedback to model the system and adjust the control law. In both indirect and direct schemes, adaptive law based upon Lyaponov quadratic stability criteria is used to adjust the weights of consequent linear state equations and consequent parameters respectively. The adaptive law ensures to withhold the plant in stable [11] condition in presence of disturbances. The controller action is composed of two terms, tracking (primary) and supervisory (auxiliary) term. The first term stems from the consequent membership function of fuzzy plant model and the second term normally from the $1^{\text {st }}$ order dynamic of sliding surface of SMC.

Typically the entire fuzzy approximated model base controllers discussed earlier utilizes Lyaponov stability criteria, for which a constant positivedefinite matrix is required to satisfy a quadratic Lyaponov function. Finding a common positive-definite matrix for large numbers of fuzzy rules or subsystem is difficult. Wang [19] proposed a technique to reduce the dimensionality of positive definite matrix, by combining fuzzy control theory with sliding mode control. In this approach nonlinear dynamics of plant is modeled and control by Mamdani-type fuzzy inference system [20] and then additional supervisory control input is designed by adaptive sliding mode control theory.

Sliding mode control (SMC) is a form of variable structure control (VSC) method, which alters the dynamics of nonlinear system by application of highfrequency switching control. SMC is simple to implement and is robust against unmodeled plant dynamics, parameter variations and disturbances [21]. With SMC the overall stability of the system is guaranteed, without finding a common positive-definite matrix to satisfy the Lyaponov quadratic criteria [7].

The SMC reduces the $n$-th order of the system to $1^{\text {st }}$ order straight sliding-switching line for which scalar Lyaponov function and simple switching control can be found easily. Large switching gains are required to force the plant states on sliding line giving rise to Bang-bang optimal control. Hence, sliding mode control describes the optimal controller for dynamic system. However the large switching gain creates "chattering" which may excite un-modeled dynamics.

To counter the problem of chattering and provide SMC with priori knowledge of upper bounds of disturbance and uncertainties $[22,23]$; adaptive sliding mode controller were proposed [24] for continuous robust action. Adaptive control can estimate the unknown parameters of the dynamic system and uses SMC to overcome unmodeled dynamics and external disturbances. However, as discussed earlier the combined adaptive SMC controller needs a linear parameterized model of the system. Fuzzy direct and indirect adaptive SMC (AFSMC) [11, 18, 25-29] schemes were then developed to fulfill the modeling requirement of adaptive SMC. In [30] robust AFSMC is proposed for nonlinear system approximated by Takagi-Sugeno (T-S) fuzzy 
model. Recently, Liang et al. [31] used T-K model for robot control, Park et al. [32] developed adaptive fuzzy SMC for the uncertain crane system incorporating fuzzy observer for uncertainties.

Another way to reduce the ambiguity and maintain consistency is to utilize expert heuristic rules, which enables the controller to be effective over the large range of parametric variation, of both plant and disturbances. As a result Procyk and Mamdani [33] proposed Self-organizing fuzzy controllers. Self-organizing fuzzy controllers (SOFC) and self-tuning fuzzy controllers (STFC) are rule-based controllers and learn on-line how to control the system [34]. The purpose of selforganizing fuzzy controller (SOFC) is to minimize the role of human experts in designing a fuzzy logic controller. In SOFC normally the input membership functions are organized. The combine action of SOFC with consequent membership function regression and adaptive weight mechanism results in self-organizing fuzzy adaptive control (SOFAC) [35]. Park et al. [16] propose SOFAC, in which rules are added to the rule based as the input space is explored. The width of the triangular membership functions is predefined and unchanged. When one of the input variable moves outside the range of the existing membership functions, a new membership function is created. Then, all the possible rules that are made available by addition of new membership functions are added to rule base. In this regard Park et al. use feedback linearization of plant and set upper bound condition of control signal gain. Phi and Gale [36] removes this condition and set the controllability condition instead. Further, Phi limits the growth and removal of membership functions.
The proposed controller in this work takes advantage of robustness and $1^{\text {st }}$ order reduction property of the SMC control [7]. There is a distinction between FSMC and sliding mode fuzzy control (SMFC). In FSMC, fuzzy control (FLC) compliments the sliding mode control in model linearization and bounding of disturbance and uncertainties. Whereas in SMFC, sliding mode complement the fuzzy control (FLC) and the fuzzy rule and stability criteria are synthesized to ensure robustness [37]. F. Song [38] describes SMFC as time suboptimal control, which has constrained Bang-bang output. Numerous AFSMC schemes have been presented in past but less attention has been paid to SMFC, except [38], further there is no report in literature on adding self-organizing scheme to the SMFC. The relationship among the different fuzzy techniques is summarized in figure 1

The proposed SOSMFC has only two level Bang-bang switching consequent membership functions, which limit the application of adaptation mechanism described earlier. Self-organizing adaptation procedure has to be adopted for antecedent membership functions adjustment and control the nonlinearities of the plant. Mudi and Pal [39] describe that FLC become adaptive; if one or more of its tunable parameter such as scaling factor, membership function and fuzzy rules are changed on-line. The control proposed here has two terms. Both terms use the same underlying principle of sliding mode controller. The primary or tracking function is carried out by a fixed parameters fuzzy sliding mode controller (FSMC) and the supervisory or auxiliary function has AFSMC. This way the stability -Lyaponov quadratic function and search of positive definite matrix is avoided. 


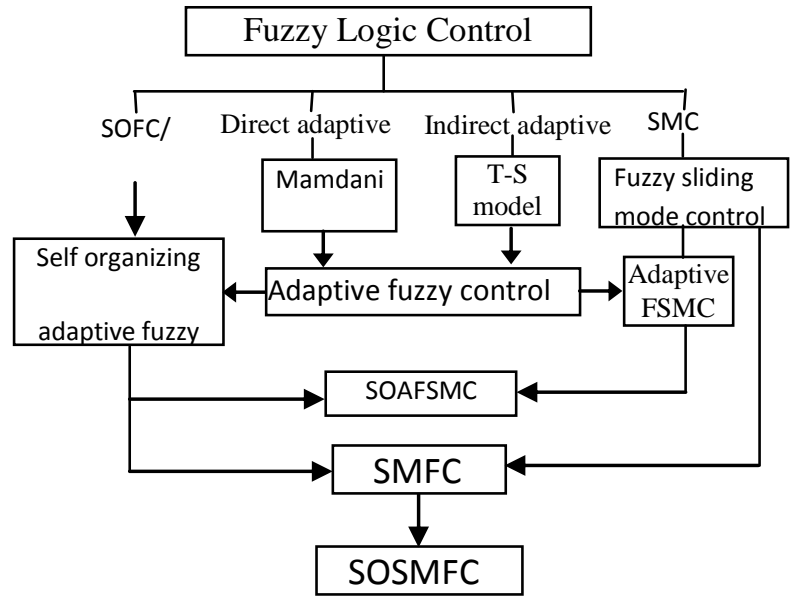

Figure 1: Relationship between different FLC Schemes

No research has been carried out on uniting the SOC and SMFC and to investigate the advantages and disadvantages of the new and combined technique has to offer. Hence, the objective of this paper is to merge together the self-organizing (SOC) technique with SMFC (SOSMFC), analyze it and compare the performance with conventional AFSMC.

The new controller works well for all non-linear problems in affine and non-affine oscillation control. The SOSMFC is based on the Mamdani model and uses largest of maxima (LOM) defuzzification technique. By arranging the output membership function in a certain way - Bang-bang output is directly obtained from the controller. The layout of this paper is first to present in section II, the theoretical background of SMC, ASFMC and SOC controllers, and then define self-organizing sliding mode fuzzy controller (SOSMFC). In section III the stability conditions of controller is described. In section IV the controller is itself developed and in section $\mathrm{V}$ the controller is simulated with benchmark problems, followed by the analysis and discussion in section VI and conclusion in section VII.

\section{NONLINEAR FUZZY CONTROLLER}

Proposed controller design preliminaries are presented here. A nonlinear model [40] will be used here for the modeling of sliding mode controller. It is shown; how the stability issues and the rule for SMFC are derived from the knowledge of sliding mode controller, followed by the development of the self-organizing sliding mode fuzzy controller.

\section{Nonlinear Model}

Consider a second order non-linear position control system [41] as

$$
M_{a} \ddot{x}+h(x, \dot{x})+l \cdot x=u(t)
$$

The Eq. (1) has the $2^{\text {nd }}$ order nonlinear form

$\dot{\mathbf{x}}=f(x, t)+b(x, t) u$

where $h(x, \dot{x})=x^{3}+0.5 x, M_{a}=0.5 \mathrm{~kg}$ and

$f(x, t)=\left[\begin{array}{c}x_{2} \\ \frac{1}{M_{a}}\left[-h\left(x_{1}, x_{2}\right)-l x_{1}\right]\end{array}\right], b(x, t)=\left[\begin{array}{c}O \\ 1 \\ \hline M_{a}\end{array}\right]$

where

$\dot{\mathbf{x}}=\left[\begin{array}{l}\dot{x}_{1} \\ \dot{x}_{2}\end{array}\right], f(x, t)=\left[\begin{array}{cc}0 & x_{2} \\ 0 & -x_{1}-2 x_{2}^{3}\end{array}\right], b(x, t)=\left[\begin{array}{l}0 \\ 1\end{array}\right]$

\section{Sliding Mode Control Rule}

Sliding - switching line $s(x, t)$ for the second order state space $\boldsymbol{R}^{2}$ is defined such that error $\mathrm{e}=x-x_{d}$, follows the line $s(x$, $t)=0$. The sliding line $s(x, t)$ is determined by

$$
s(x, t)=\left(\frac{d}{d t}+\lambda\right)^{n-1} e
$$

Eq. (3) can be expanded with binomial expansion and $\lambda$ is positive constant. For $n=2$

$$
\mathrm{s}=\dot{e}+\lambda e \quad \because \dot{e}=\dot{x}-\dot{x}_{d}
$$


then from Eqs. (2) and (4)

$$
\dot{s}=f(x, t)+b(x, t) u+\lambda \dot{e}-\ddot{x_{d}}
$$

Multiplying it with $s$ yields

$$
s \dot{s}=f(x, t) s+b(x, t) u s+\lambda \dot{e} s-s \ddot{x}_{d}
$$

The control value $u$ should be selected so that for $b>0, \dot{s i}<0$. The slope of sliding line is represented by $\lambda$.

$$
s \dot{s}=\left\{\begin{array}{llll}
< & 0 & \text { for } & s<0, \text { increasing } u \\
= & 0 & \text { for } & s=0 \\
< & 0 & \text { for } & s<0, \text { decreasing } u
\end{array}\right.
$$

These simple implications will become the building blocks of fuzzy rules for SMFC.

\section{Sliding Mode Control Law}

In general, $f(x, t)$ is not precisely known, but upper bounded by a known continuous function of $x$. Similarly $b(x, t)$ is not known, but is of known sign and is bounded by a known continuous function of $\mathbf{x}$ as

$$
\begin{aligned}
& |f-\hat{f}| \leq F(x ; t) \\
& \frac{1}{\beta(x ; t)} \leq \frac{\hat{b}}{b} \leq \beta(x ; t)
\end{aligned}
$$

Where $\hat{f}$ and $\hat{b}$ are estimated values of $f$ and $b$ respectively, without the function argument for brevity purpose.

\section{SMC Equivalent Control}

Let $u_{e q}$ be the equivalent control law that will keep the states on the sliding trajectory, computed by $\dot{s}=0$ for $u \equiv u_{e q}$, replacing $\lambda$ by input gain vector $\boldsymbol{g}=\left[\begin{array}{ll}g_{1} & g_{0}\end{array}\right]$ and from Eqs. (3) and (4)

$$
\begin{aligned}
& s=g_{1} \dot{e}+g_{0} e \\
& \dot{s}=g_{1} \ddot{e}+g_{0} \dot{e}
\end{aligned}
$$

From Eq. (5) with estimated uncertainties

$\left.\dot{s}\right|_{u=u_{e q}}=\hat{f}(x ; t)+\hat{b}(x, t) u_{e q}-\ddot{x}_{d}+\mathrm{g}^{\mathrm{T}} \mathrm{e}=0$

Solving the above equation

$$
u_{e q}=\frac{1}{\hat{b}}\left[-\hat{f}(x ; t)+\ddot{x}_{d}-\boldsymbol{g}^{\boldsymbol{T}} \boldsymbol{e}\right]
$$

Let

$\hat{u}=\left[-\hat{f}(x ; t)+\ddot{x}_{d}-\boldsymbol{g}^{T} \boldsymbol{e}\right]$

$\boldsymbol{g}^{\boldsymbol{T}} \boldsymbol{e}=\left[\begin{array}{ll}g_{1} & g_{0}\end{array}\right]\left[\begin{array}{l}\dot{e} \\ e\end{array}\right]$

The control input $u_{e q}$ keeps the error states on sliding surface $\dot{s}=0$. The estimate $\hat{f}(x ; t)$ of the system is approximated by sliding mode fuzzy controller output $u_{f}$ under the assumption 1.

Assumption 1: The nonlinearities of the plant vanishes on the sliding surface $\mathrm{s}=0$.

\section{SMC Reaching Control}

The control input $u_{s}$ to get the state $x(0)$ to reach $x_{d}$ is then made to satisfy the Lyapunov-like function $V=(1 / 2) s^{2}$, if there exist $\eta>0$ and by the following sliding condition :

$$
\frac{1}{2} \frac{d}{d t} s^{2}(x, t) \leq-\eta|s|
$$

This control law ensures that the system trajectories from initial state to desired state or $\dot{s}=0$, reduces in squared distance term. In conventional notation Eq. (11) is written as

$$
\begin{aligned}
& \dot{s} . s \leq-\eta|s| \quad \eta>0 \\
& \text { or } \\
& \dot{s} \cdot \operatorname{sgn}(s) \leq-\eta
\end{aligned}
$$

The complete sliding mode control law $u$ then becomes 
$u=\left[u_{e q} \wedge u_{S}\right]$

where from [7]

$u_{s}=-K \operatorname{sgn}(s)$

and

$\operatorname{sgn}(s)= \begin{cases}+1, & \text { if } s>0 \\ -1, & \text { if } s<0\end{cases}$

Using a sign function often causes chattering in practice. One solution is to introduce a boundary layer around the switch surface [7]. A continuous consequent output, a small deadband $\Delta \mathrm{s}= \pm 0.001$ is included in output rendering the control action Bang-off-bang. Resulting in switching control

$u_{s}=\left\{\begin{array}{l}+M, \quad \text { if } s>0 \\ 0 \quad \text { if } \quad \Delta s= \pm 0.001 \\ -M, \quad \text { if } s<0\end{array}\right.$

where $M$ is a constant. SMC reduces the order of the system to $1^{\text {st }}$ order straight sliding-switching line for which, as shown above scalar Lyaponov function and simple switching can be found easily. The control rules Eq. (15) will be incorporated in SMFC to give rise to Bang-bang optimal control.

\section{NONLINEAR CONTROLLER AND STABILITY}

SMC controllers have been developed and applied to nonlinear systems for the last two decades. In the absence of a mathematical model for non-linear fuzzy controllers the stability analysis is not straight forward, whereas the stability of SMC is inherent. SMC is a robust control method [7] and its stability is proven with Lyapunov's direct method [12]. Wong et.al [40] presents a method to apply Lyapunov's direct method to individual firing rules rather than the entire fuzzy sub-system associated with all the rules.

Lyaponov theorem 1: Assume that there exists a scalar function $V$ of the state $x$ such that

i) $V=x^{T} P x$ is positive definite-and continuously differentiable, $\mathrm{P}$ is square positive definite matrix

ii) $\dot{V}$ is negative definite in the active region of the corresponding fuzzy rule

From model Eq. (2) by letting $\mathrm{s}=a \cdot x_{1}+x_{2}$, where $\mathrm{a}=1$, the slope of the sliding line.

$$
\begin{aligned}
& s=x_{1}+x_{2} \\
& =x_{2}-2 x_{2}^{3}-x_{1}+u
\end{aligned}
$$

The Lyapunov's stability function Eq. (11) is selected as

$$
\begin{aligned}
& V=\frac{1}{2} s^{2} \\
& \dot{V}=s \dot{s} \\
& \dot{V}=s\left(x_{2}\left(1-2 x_{2}^{2}\right)-x_{1}+u\right)
\end{aligned}
$$

For $V$ to be negative definite then

$$
x_{2}\left(1-2 x_{2}^{2}\right)-x_{1}+u=\left\{\begin{array}{cccc}
< & 0 & \text { for } & s>0 \\
= & 0 & \text { for } & s=0 \\
> & 0 & \text { for } & s<0
\end{array}\right.
$$

Let $\beta(x)=-\left(x_{2}\left(1-2 x_{2}^{2}\right)-x_{1}\right)$

Then for negative definite $\dot{V}$ (see Eq. (18)) the following $u$ is required

$$
u=\left\{\begin{array}{ccc}
\dot{V}=(s<0)(-\beta(x)+u) \Rightarrow & \text { stable } & \text { if } u>\beta(x) \\
\dot{V}=(s=0)(-\beta(x)+u) \Rightarrow & 0 & \text { if } u=\beta(x) \\
\dot{V}=(s>0)(-\beta(x)+u) \Rightarrow & \text { stable } & \text { if } u<\beta(x)
\end{array}\right.
$$


The following control law ensures $V$ is negative

$$
u=\beta(x)-k . \operatorname{sign}(s)
$$

where $k>0$.

The stability of SMC for Eq. (2) be verified by using Lyapunov's stability test [40]. Selecting a Lyaponov function

$$
\begin{aligned}
V & =\overline{x^{T}}\left[\begin{array}{cc}
10 & 1 \\
1 & 10
\end{array}\right] \bar{x} \\
& =10 x_{1}^{2}+2 x_{1} x_{2}+10 x_{2}^{2}
\end{aligned}
$$$$
\text { If } x \in[-2,2] \text { and }
$$

$x d o t \in[-2,2]$ then $y=-M$,

$$
\begin{aligned}
\dot{V} & =20 x_{1} \dot{x}_{1}+2 \dot{x}_{1} x_{2}+2 x_{1} \dot{x}_{2}+20 x_{2} \dot{x}_{2} \\
& =20 x \dot{x}+20 \dot{x} \dot{x}+2 \dot{x}^{2}+2 x \ddot{x} \\
& =20 x \dot{x}+20 \dot{x}\left(-2 \dot{x}^{3}-x-M\right)+2 \dot{x}^{2}+2 x\left(-2 \dot{x}^{3}-x-M\right) \\
& =20 x \dot{x}-40 \dot{x}^{4}-20 \dot{x} x-20 \dot{x} M+2 \dot{x}^{2}-4 \dot{x}^{3} x-2 x^{2}-2 x M \\
& =-2 M(10 \dot{x}-x)-40 \dot{x}^{4}+2 \dot{x}^{2}-4 \dot{x}^{3} x-2 x^{2} \\
& \leq O
\end{aligned}
$$

if $x \in[-2,2]$ and $x d o t \in[-2,2]$ then $\mathrm{y}=$ $+\mathrm{M}$

results in $\dot{V} \leq 0$

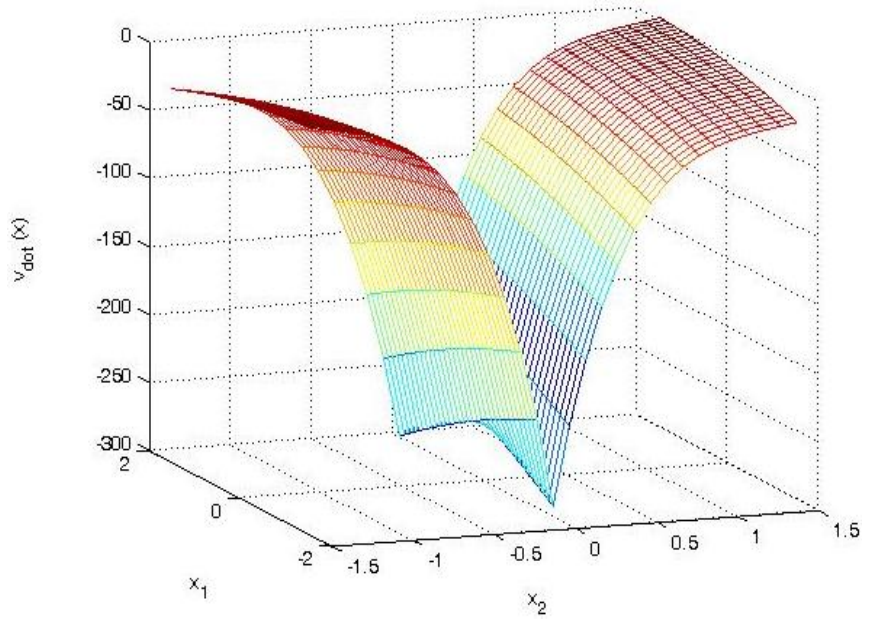

Figure 2: Negative Definite $V(x)$ Surface for Optimal Rules in Table 1, Eq. (18)
Complete set of fuzzy sub-systems surface to establish stability of two level Bang-bang output $u=(-1,+1)$ is shown in Figure 3 .

\section{NONLINEAR FUZZY BANG-BANG CONROLLER}

The new proposed controller combines the fuzzy logic inputs with a relay output in a single entity. Due to fact that the proposed controller has fuzzy rules based on sliding mode control theory and its has a self-organizing membership function capability on its antecedent, it is called as self-organizing sliding mode fuzzy controller (SOSMFC). The SOSMFC uses Largest of maxima (LOM) defuzzification technique to yield a bang-bang output.

In fuzzy controller, it is necessary to specify the universe of discourse for the states of the system, which are considered to be a reasonable representation of all the situations that the controller may face. In the self-organizing controller presented here the universe of discourse (UOD) $\mathrm{Y}_{i}$ is a function of states, $i$ is the input index of the controller. Then for SISO system in eq. (2) the universe of discourse

$$
\mathrm{Y}_{\mathrm{i}}(t)=\max \left[-x_{i, O}(t), x_{i, O}(t)\right]
$$

\section{Fuzzzy Input Membership Functions Set}

The state $\mathrm{x}$ is used for selection of self-organizing and tuning of supervisory control $u_{s}$. Depending upon the initial state $x_{0}$ the controller select the appropriate number of membership functions and then start tuning the input gain, until a error condition $e=\varepsilon( \pm 0.001)$ is reached and the control is transferred to fixed parameter control action, $u_{e q}$. The input variable $i$ and the value assigned to fuzzy set of triangular membership functions are based on mathematical characteristic, Table 1. 
$A_{i=1,2}^{k=1 \ldots K}$, is the linguistic label assigned to each $\mathrm{mf}$, and input fuzzy set for selforganizing controller is shown in Figure 2. The index $k \ldots . K$, is the range of $K$ number of membership functions. $\mu_{A_{i}{ }^{k}}$, represent the degree of the membership functions. New membership functions $A_{i}^{k}$ are added in pairs on either side to balance the fuzzification process, as shown by matching styles of lines.

Initially a set of three membership functions is used $(K / 2)+1,(K / 2)+2(K / 2)+3$ as default membership functions $A_{i}^{(K / 2)+1}, A_{i}^{(K / 2)+2}, A_{i}^{(K / 2)+3}$, shown in thick black line in figure 2 . Odd and symmetrical arrangement of membership functions ensures the balance output around ' $O$ ' and removes biasness during the defuzzification process. Additions of membership functions is simply governed by comparing the current state $x_{i, \text { new }}(t), x_{i}(0)=x_{0}$, with previous $\max$ values of $x_{i, \text { old }}(t)$

$$
x_{i, \text { new }}(t) \geq \sup \left[x_{i, \text { old }}(t)\right]
$$

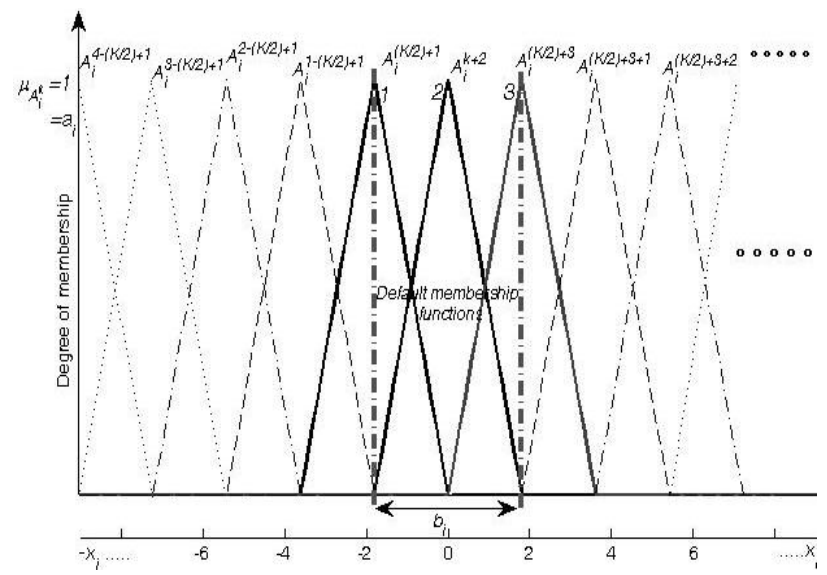

Figure 2: Self-Organizing Input Fuzzy Membership Function for State $x$ Controller

If the above condition is true then a index $\Delta$ is incremented by one and $A_{i}^{\Delta-(K / 2)+1}, A_{i}^{(K / 2)+3+\Delta}$ are incremented in pair on both sides. The $\Delta$-step is kept incremented until the Eq. (21) conditions of self-organizing fails at $K$

$$
\begin{gathered}
\Delta=\Delta+1 \\
K=\mid 2 * \text { floor }\left[\frac{x_{i} / 2}{\Delta}\right]+1 \mid \\
\quad \mathbf{Y}_{\mathrm{i}, \text { new }} \geq \mathrm{Y}_{\mathrm{i}, \text { old }}
\end{gathered}
$$

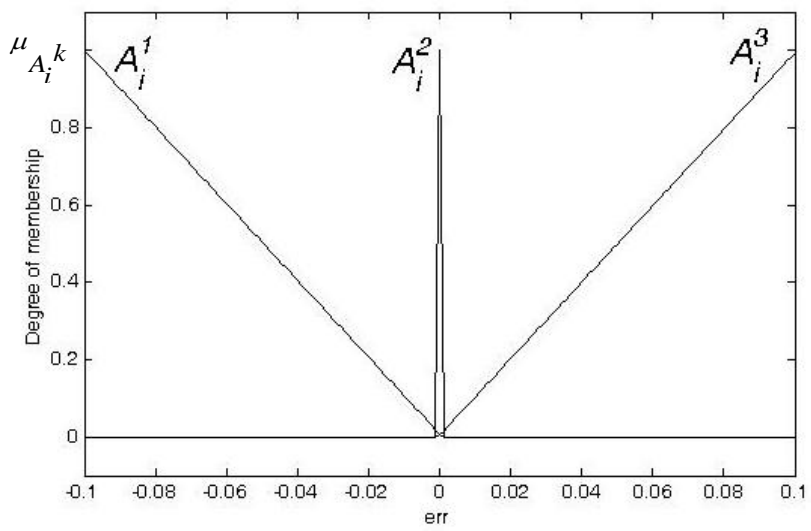

Figure 3: Input mf for Fixed Tracking Control Signal

\section{Fuzzy Output Membership Function Set}

The output membership function has largest of maxima output (LOM) [41], which emulates hardware relay bi-level Bang-bang output, as shown in figure 4. $\Psi_{i}$ $=[-M, M]$ is the output universe of the discourse. The upper bound of $M$ is defined as $M_{d}$

$$
M_{d} \leq \max |\hat{f}(x, t)|
$$

The gain $M_{d}$ can be set during the run time. In figure 4 a small deadband $\Delta s= \pm 0.001$ is set by $\mathrm{mf}$ ' $Z$ ' is shown, which filter out the high frequency chattering; associated with Bang-bang output on the sliding line.

\section{Fuzzy Process and Rules}

Fuzzification between the two inputs $e$ and $\dot{e}$ is an minimum inference for the $j^{\text {th }}$ rule described as 


$$
\mu(y)_{B_{j}}=\left[\mu(e)_{A_{1, j}^{k}} \wedge \mu(\dot{e})_{A_{2, j}^{k}}\right]
$$

The sliding mode based fuzzy rules are given Table 1. If $A_{1}^{1}--v e$ and $A_{2}^{2}$ is +ve then $u=+M$

If $A_{1}^{3}$-ve and $A_{2}^{3}$ is +ve then u =Zero

If $A_{1}^{K}++$ ve and $A_{2}^{3}$ is 0 then $u=-M$

Table 1: Sliding Mode Control Based Fuzzy Control

\begin{tabular}{|c|c|c|c|c|c|}
\hline e dot: & $--A_{1}{ }^{1}$ & $-A_{1}{ }^{2}$ & $0 \mathrm{~A}_{1}{ }^{3}$ & $+A_{1}{ }^{4}$ & $++A_{1}{ }^{K}$ \\
\hline$++A_{2}{ }^{1}$ & & $-M$ & $-M$ & $-M$ & $-M$ \\
\hline$+A_{2}{ }^{2}$ & $+M$ & & $-M$ & $-M$ & $-M$ \\
\hline $0 A_{2}{ }^{3}$ & $+M$ & $+M$ & & $-M$ & $-M$ \\
\hline$-\mathrm{A}_{2}{ }^{4}$ & $+\mathrm{M}$ & $+M$ & $+\mathrm{M}$ & & $-M$ \\
\hline$-A_{2}{ }^{K}$ & $+\mathrm{M}$ & $+M$ & $+M$ & $+M$ & \\
\hline
\end{tabular}

Theorem 1. The sign of output $M$, of control law Eq. (13) is decided by the negative of sum of the inputs of the $j^{\text {th }}$ rule as

$$
u_{f_{j}}=-1 * M * \operatorname{sign}\left[A_{1, j}^{k}+A_{2, j}^{k}\right]
$$

Corollary. In rule: If $A_{1}^{1}--v e$ and $A_{2}^{2}$ is +ve, then the negativity of ' $A_{l}^{l}$ ', is higher than the positivity of ' $A_{2}^{2}$, resulting in an opposite sign control signal of $+M$, Eq. (25). This steers the state toward the sliding surface. If two inputs membership functions magnitude is equal, then $M=$ Zero.

The minimum inference in Eq. (23) for $j^{\text {th }}$ rules are aggregated by largest of maxima (LOM) as

$$
\begin{aligned}
& \sigma_{f}(y)=\left[\mu(y)_{B_{1, j}} \vee \mu(y)_{B_{2, j}} \vee \ldots . . \mu(y)_{B_{i, j}}\right] \\
& \sigma_{f}(y)=\max \left[\mu(y)_{B_{l, j}}\right]
\end{aligned}
$$

Changing the dependency to $e$, then from Eq. (24) and Eq. (26)

$$
\sigma_{f}(e)=\max \left[\mu(e)_{A_{1, j}^{k}} \wedge \mu(\dot{e})_{A_{2, j}^{k}}\right]
$$

The Bang-bang supervisory switching control based on (27) produces control signal (25) based upon LOM defuzzification

$$
u_{s}=M * \operatorname{sgn}\left[\sigma_{f}(e)\right]
$$

Then from Theorem 1 and Eq. (28)

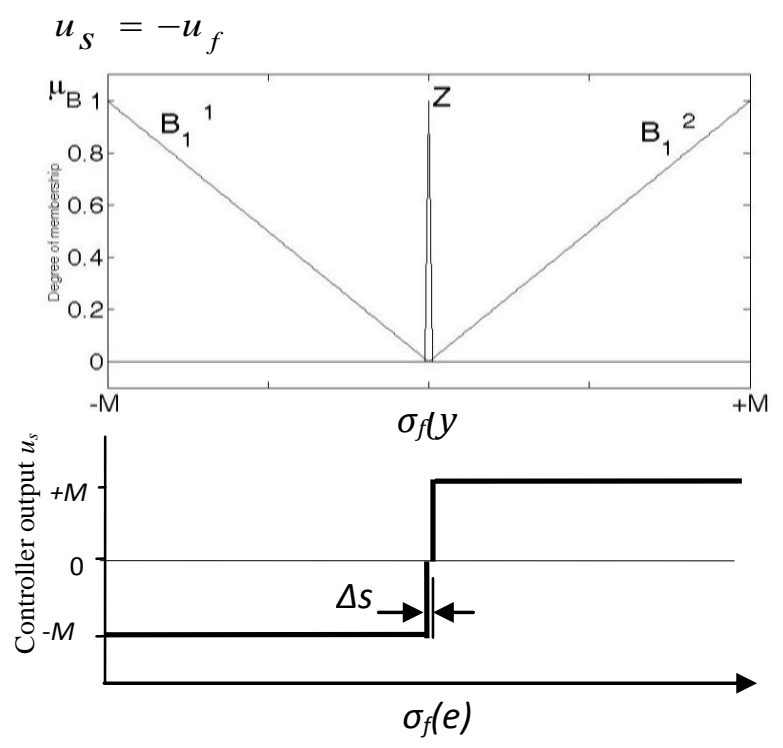

Figure 4: (Top) SOSMFC output $y$ membership functions. (Bottom) two levels Bang-bang control output $u_{\mathrm{s}}$

Largest-of-Maximum (LOM) uses the union of the fuzzy sets and takes the largest value 
of the domain with maximal membership degree. The output membership functions are shown in Figure 4. The output of SOSMFC depends upon the maximum value of degree of member-ship function, shown in Figure 4 (Top).

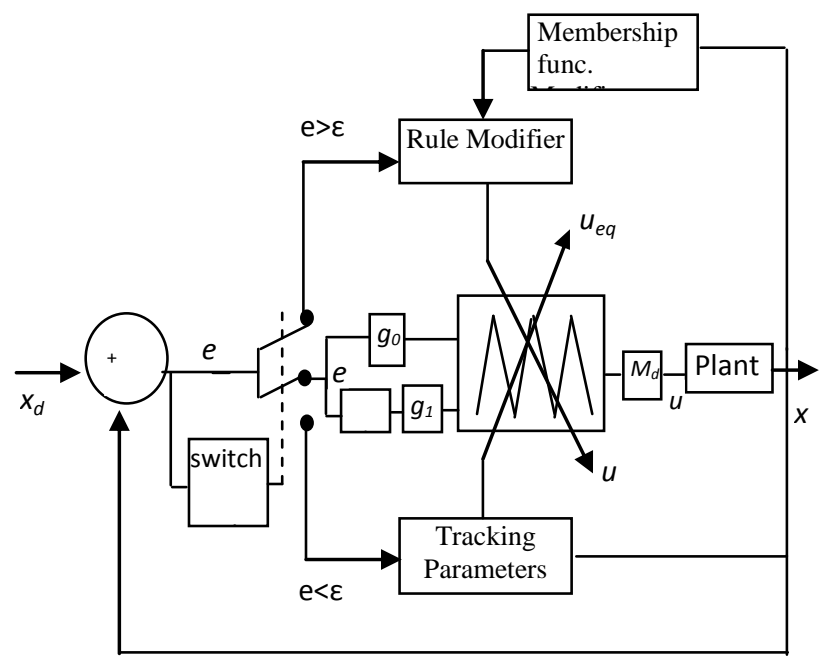

Figure 5: Block Diagram of Self Organizing Sliding Mode Fuzzy Controller (SOSMFC)

The controller has the same SMFC structure for both $u_{e q}$ and $u_{s}$ control action. The control action $u$ (13) and SOSMFC scheme used in the paper is shown in figure 5. Depending upon initial condition $x_{o}$ and $\mathrm{e}$ $>\varepsilon$, where $\varepsilon=0.001$, the hitting control $u_{s}$ acts first and the membership function modifier block selects and ensembles the required number of membership functions to enclose the state $x$, eqs. (20), (22), figure 2 . The rule modifier, increase the rules automatically according to rule table 2 for as many membership function as requested by the membership modifier. At the end of hitting condition the control is transferred to the tracking controller which ensures that the output $x$ follows the desired input $x_{d}$.

\section{Self -Tuning Control}

For sliding mode fuzzy control Eq. (10), e and $\dot{e}$ has the gains $g_{0}<1$ and $g_{1}<1$, Figure 4 . Which has a effect of proportional and derivative gain [43] on the inputs. According to Passino [43] under these conditions the inputs $e$ and $\dot{e}$ has inverse scaling factor effect on the horizontal axis resulting in reduced inputs, which converge toward the central membership function. This increase the Bang-bang switching activity around the central membership function $A_{i}^{2}$, in -ve and +ve halves, Figure 3.

The tuning is used during the supervisory control $u_{s}$ and is based on the current state $x$, having universe of discourse $\mathrm{Y}_{i}=\left[-x_{i}, x_{i}\right]$. As the error $e$ and $\dot{e}$ reduce so does value of the state $x$. Again according to Passino [43] the inputs will see this as contraction and the input moves away from the central membership function which eases the switching-chattering effect as error and states values becomes smaller.

\section{Operational Details of SOSMFC}

The details of controller operation are illustrated in Figure 6 for system Wong [40]. For initial condition $x_{i}(0)=20$, the self-organizing is completed in three steps. In step one $\Delta=1$ and three defaults membership functions shown in figure 2 are shown at left in Figure 6. With this arrangement the controller in figure 4 is unable to reduce the state $x$ and condition in Eq. (21) is satisfied. As a result, step two in initiated and $\Delta$ in incremented by 1 to $\Delta=2$, and two more membership functions are added to existing three $(3+2=5)$, second set of membership functions in Figure 6 is second from left. With new mf setting the controller continues to satisfy the condition of self-organizing in Eq. (21) and step three $\Delta=3$ is invoked again, and the membership functions increase to $K=7$, set of seven membership function is shown in Figure 6.

$$
\begin{aligned}
K(\Delta) & =\mid 2 * \text { floor }\left[\frac{20 / 2}{3}\right]+1 \mid \\
K(\Delta) & =2 * 3+1 \\
& =7
\end{aligned}
$$




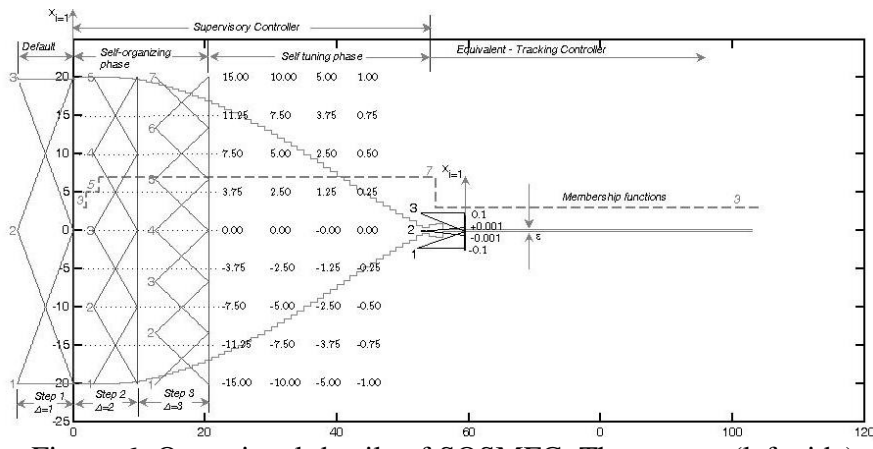

Figure 6: Operational details of SOSMFC. Three steps (left-side) are shown during the self organizing phase. Dash line shows the membership functions status during the operation. Note the tuning is done on $\mathrm{K}$ number of mf, eq.(22).

\section{BENCH MARK PROBLEMS SIMULATION}

To prove the effectiveness of the SOSMFC, five benchmark SISO problems including non-affine nonlinear and duffing oscillation problems are selected. In these problems the initial condition are exaggerated to show the regulation and tracking ability of the controller.

\section{Second Order Non-Linear Problem}

The first, a second order nonlinear problem in Wong [40], eqs (1-2), $x_{d}=2 \sin (t)$ $+\cos (0.5 t)$, is

$$
\ddot{x}(t)+x^{3}+0.5 x(t)+0.5 x(t)=u(t)
$$

The SMC and stability of this problem has been discussed in section III. The controller rules are based on Table 2. The control signal Eq. (25) under the scheme shown in figure 5 is used. The output gain $M_{d}$ is given in Eq. (23).

\section{First Order Nonlinear Plant}

This example appears in Wang [11] and is unstable without the control. The desired control is to regulate and track a reference trajectory, $x_{d}=2 \sin (t)+\cos (0.5 t)$

$$
\dot{x}(t)=\frac{1-e^{-x(t)}}{1+e^{-x(t)}}+u(t)
$$

\section{Chaotic System}

Duffing forced oscillation in Wang

[11]

$$
\begin{aligned}
& x_{1}=x_{2} \\
& \dot{x}_{2}=-0.1 x_{2}-x_{1}^{3}+12 \cos (t)+b u(t)+d(t)
\end{aligned}
$$

Tracks the desired oscillation $x_{d}=\sin (1.5 t)$ and $d(t)=0.1 \operatorname{rand}(1,20)$

\section{Second Order Non-Linear Problem}

The problem is reference in Tanaka [12] and the output tracks $x_{d}=\cos (0.5 t)+$ $\sin (t)$

$$
\ddot{x}(t)=0.1 x^{3}-0.02 x(t)-0.67 x^{3}(t)+u(t)
$$

\section{Nonlinear Servo Mechanism} [42].

The problem appears in Kovacic

$$
\begin{aligned}
& \dot{x}_{1}=x_{2} \\
& \dot{x_{2}}=-x_{2}-0.4 \sin \left(x_{1}\right)+d(t)+u(t)
\end{aligned}
$$

The desired is $x_{d}=(\pi / 3) \sin (0.1 t)$ and $d(t)=2 \cos (0.1 t)$
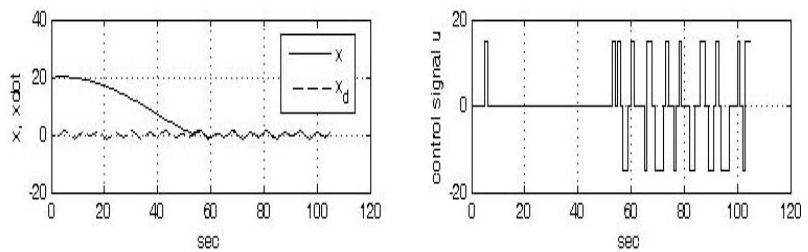

Figure 7: Bench Mark Problem A, Wong [40] $x(0)=20, \quad x \operatorname{dot}(0)=0, \quad M_{d}= \pm 15$. The SelfOrganizing and Tuning Detail are Given in Figure 5

\section{Non-Affine Nonlinear Problem}

The non-affine system is described in Park [16] 
$x_{1}=x_{2}$

$\dot{x_{2}}=x_{1}^{2}+0.15 u^{3}+0.1\left(1+x_{2}^{2}\right) u+\sin (0.1 u)+d(t)$

The desired tracking trajectory is $x_{d}=$ $\sin (t)+\cos (0.5 t)$ and $d(t)=0.5 \sin (10 t)$
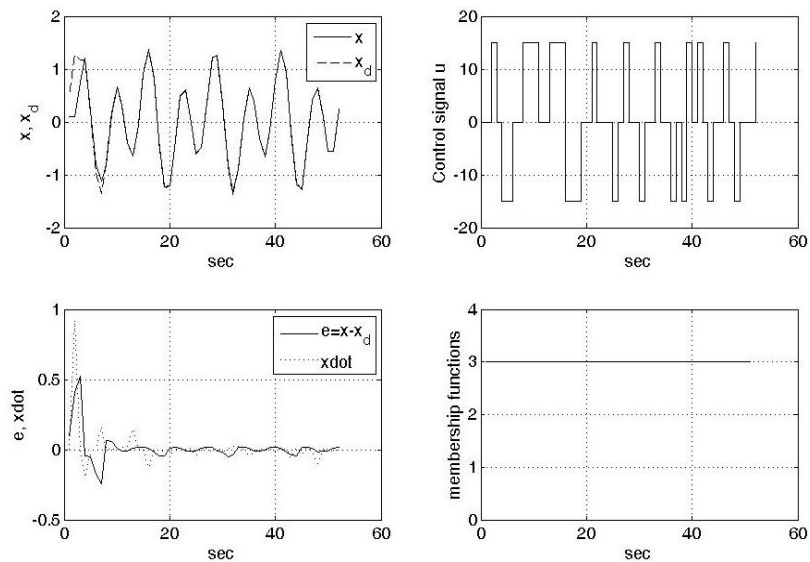

Figure 8: Bench Mark Problem A, Wong [40] $x(0)=$ $x \operatorname{dot}(0)=0, M_{d}= \pm 15$. The Controller Requires SelfTuning Only and Doesn't Need Any Additional Membership, which Remains at Three
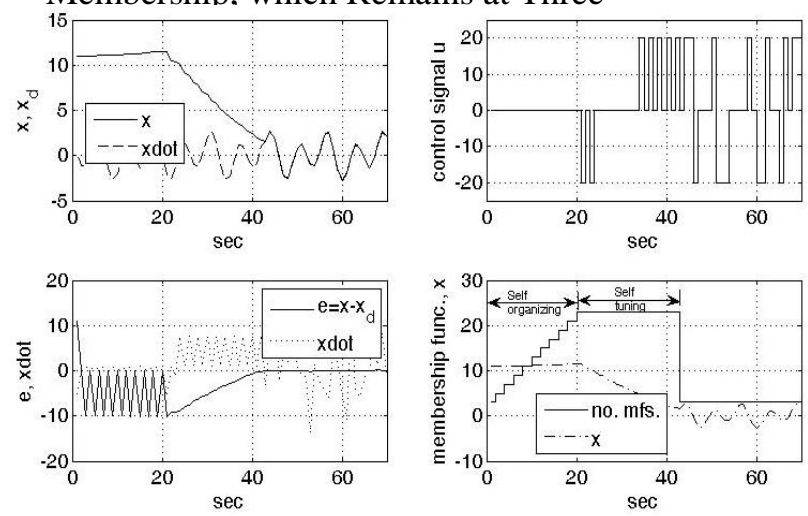

Figure 9: Bench Mark Problem B, Wang [11]. $x(0)=7, x \operatorname{dot}(0)=-5$. Bottom Right Figure Shows the Self Organizing and Tuning Operation of the Controller
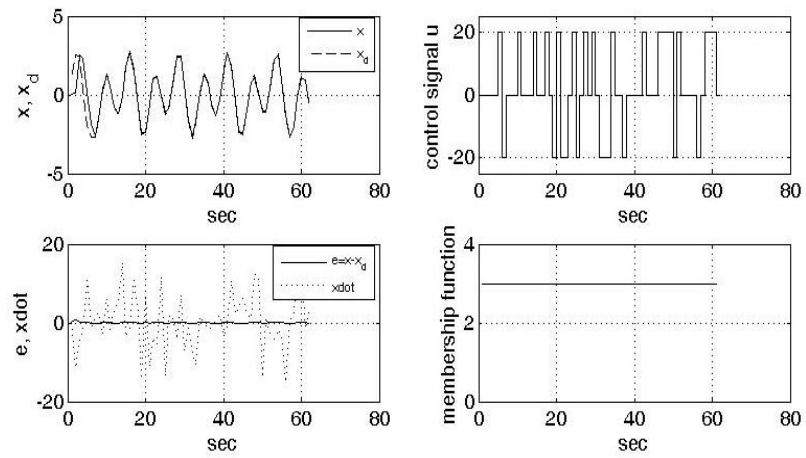

Figure 10: Bench Mark problem B, Wang [11], $x(0)=0, x \operatorname{dot}(0)=0$. Bottom right figure shows only tuning .operation of the controller
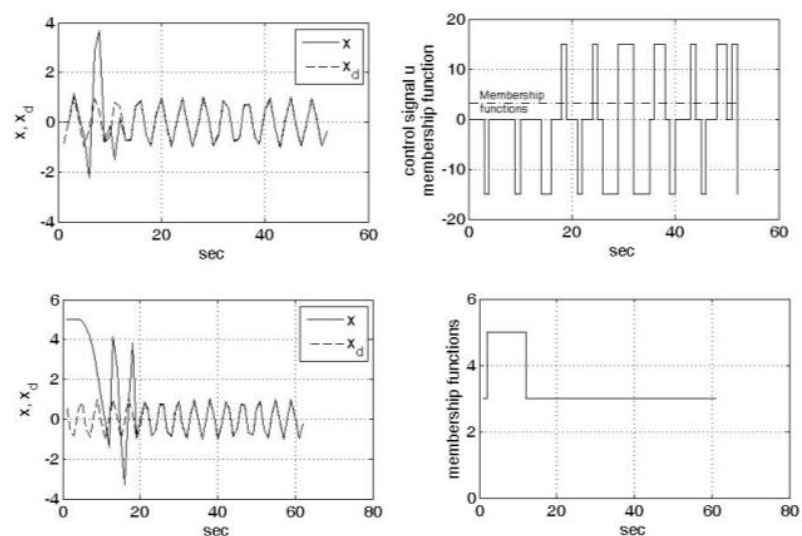

Figure 11: Duffing Oscillation-Bench Mark Problem C, Top: $x(0)=0$ and $\mathrm{mfs}=3$. Bottom $\mathrm{x}(0)=5$ and $\mathrm{mfs} 3-5-3$
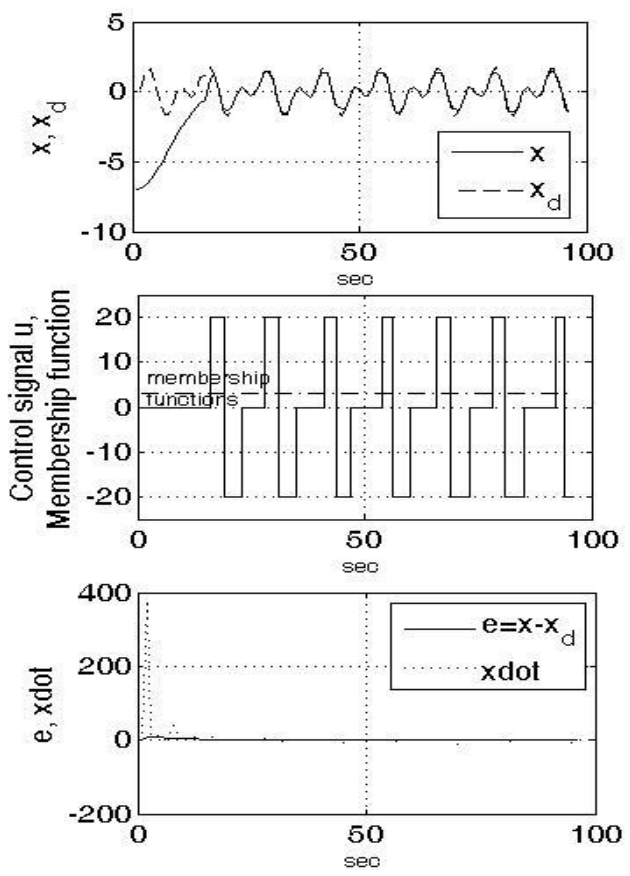

Figure 12: Bench mark problem D. Tanaka [12], $\mathrm{x}(0)=-7, \mathrm{xdot}(0)=-2, \mathrm{~g}_{0}=\mathrm{g}_{1}=0.1$ and $\mathrm{M}_{\mathrm{d}}=20$

\section{SIMULATION RESULTS}

Six most referenced SISO benchmark problem appearing in adaptive fuzzy control research publications are tested to evaluate the performance of the proposed controller.

The controller capability as a regulator and tracking controller is proven by successfully 
resetting the large initial states $x$. The simulation results in figures 7-15, shows the states $x, x d o t$, desired signal $x_{d}$, error, the control signal $u=u_{s}+u_{e q}$, and self organizing count of additional membership required by the controller to converge to desired signal. $M_{d} \leq 20$ for all the problems demonstrated, smaller value of $M_{d}$ may not be sufficient to drive the system to sliding mode surface. $M_{d}$ depends upon the upper bond $f^{U}$ of $f(x, t)$ and uncertainties. In default mode and tracking phase the number of membership functions is three. In tracking phase the membership functions are fixed and have a small deadband. It can be observed that controller uses selforganizing membership functions adjustment - if the initial state has large value. Higher initial condition can be used for the examples with higher values of $M_{d}$.
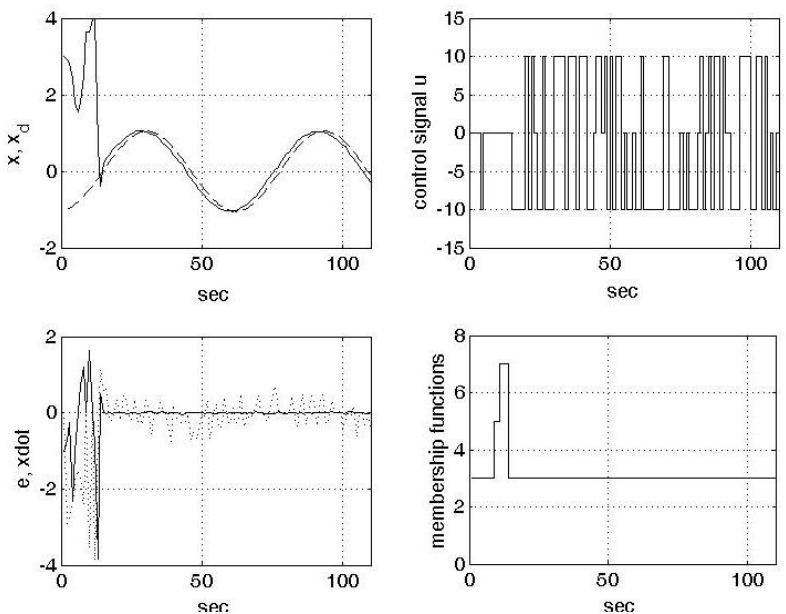

Figure 13: Bench Mark problem E, Kovacic [42], $\mathrm{x}(0)=3, \mathrm{xdot}(0)=-1$, Gains $\mathrm{g}_{0}=\mathrm{g}_{1}=0.1$, $\mathrm{M}_{\mathrm{d}}=10$
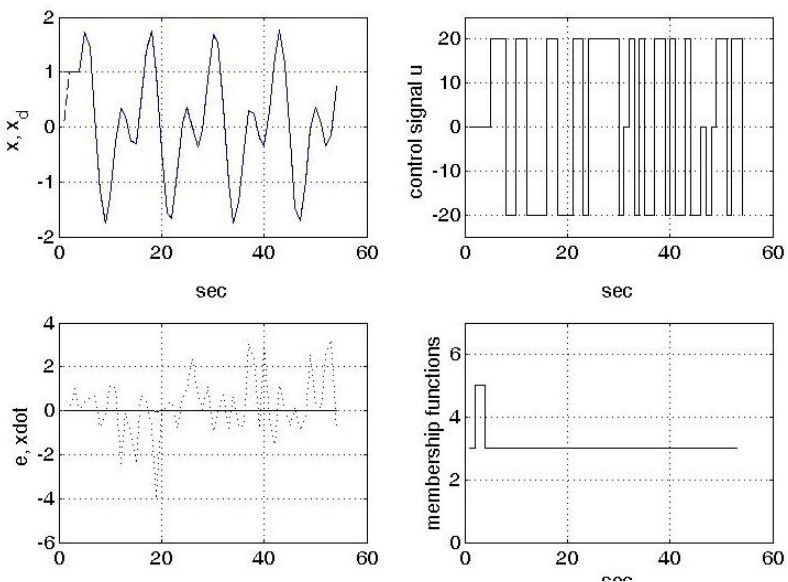

Figure 14: Bench Mark problem F, Park [16], $\mathrm{x}(0)=1, \mathrm{x} \operatorname{dot}(0)=0$. Gains $\mathrm{g}_{0}=0.1, \mathrm{~g}_{1}=0.001, \mathrm{M}_{\mathrm{d}}=20$
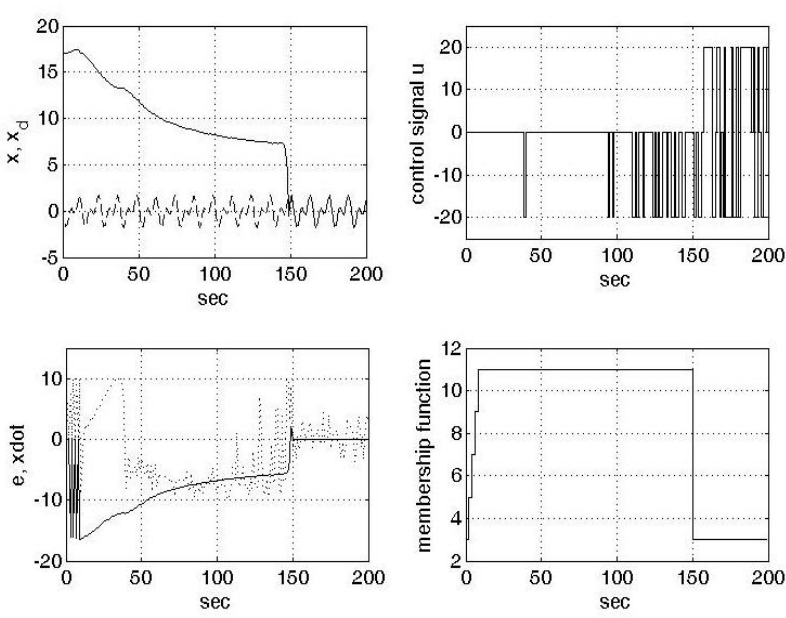

Figure 15: Bench Mark problem F, Park [16], $\mathrm{x}(0)=17, \quad \mathrm{xdot}(0)=6 . \quad$ Gains $\quad \mathrm{g}_{0}=0.1, \quad \mathrm{~g}_{1}=0.001$, $\mathrm{M}_{\mathrm{d}}=20$

\section{CONCLUSION}

The new controller works as an adaptive sliding mode fuzzy controller and further has capability of regulating large initial states. The controller uses sliding mode principle and stability criteria for reduced order system to simplify the nonlinear dynamics of the system. Only input membership functions are added or tuned in supervisory phase and fixed tracking controller parameters are used in equivalent control phase. Unlike conventional $\mathrm{T}-\mathrm{S}$ based adaptive fuzzy controllers, where positive definite matrix is required for Lyaponov stability criteria to find weights for fuzzy basis and adaptive law. The proposed controller uses fuzzy rule based on SMC $-1^{\text {st }}$ order Lyaponov criteria to satisfy the stability criteria. The controller has a simple configuration and is easy to implement.

\section{REFEENCES}

[1] A. Isidori, Nonlinear Control Systems: An Introduction. New York: Springer, 1989. 
[2] S. N. Singh, "Decoupling of invertible nonlinear system with state feedback and pre-compensation," IEEE Trans. Automat. Control, vol. 25, no 6, pp.1237-1239, 1980

[3] P.J. King and E. P. Mamdani, " The Application of fuzzy control systems to industrial process, " Automatica, vol. 13, pp. 235-242, 1977

[4] T. Takagi and M. Sugeno, “ Identification of Systems and its application to modeling and control," IEEE Trans. on System, Man and Cybernetics, vol. 15, pp. 116-132, 1985.

[5] S. F. Su and K. Y. Chen, "Fuzzy hierarchical data fusion networks for terrain location identification problem," IEEE Trans. on System, Man and Cybernetics Part B, vol. 34, no. 1, pp. 731-739, 2004.

[6] S. F. Su and S. R. Huang, "Applications of model-free estimators to the stock market with the use of technical indicators and nondeterministic features," Journal of the Chinese Institute of Engineers, vol. 26, no. 1, pp. 21-36, 2003.

[7] Slotine J. J. E and Weiping Li, Applied Nonlinear Control, Prentice Hall, New Jersey, 1991

[8] Jin L., Nikiforuk P. N. and Gupta M. M., "Fast Neural Learning and Control of Discrete-Time Nonlinear Systems," IEEE Trans. on System, Man and Cybernetics vol. 25, no. 3, pp. 478-488, March 2000.

[9] Li-Xin Wang, "Fuzzy systems are universal approximators," Proc. IEEE Int. Conf. Fuzzy Systems(San Diego). 1992, pp. 1163-1170

[10] J. L. Castro, "Fuzzy Logic Controllers Are Universal Approximators," IEEE Trans. on System, Man and Cybernetics vol. 25, no. 4, pp. 629-653, April 1995.

[11] Li-Xin Wang, "Stable Adaptive Fuzzy Control of Nonlinear Systems," IEEE
Trans. on Fuzzy Systems. Vol. 1, no. 2, pp. 146-155, May 1993.

[12] T. Takagi and M. Sugeno, "Stability analysis and design of fuzzy control system," Fuzzy Sets System, vol. 45, no. 2, pp. 135-156, Jan. 1992.

[13] L. X. Wang, "Stable adaptive controllers with application to inverted pendulum tracking,' IEEE Trans. on System, Man and Cybernetics vol. 26, no. 5, pp. 677-690, 1996.

[14] N. Essounbouli and A. Hamzaoui, "Direct and Indirect Adaptive Fuzzy Controllers for a Class of Nonlinear5r Systems," Intl Jour. of Automation and Systems, Vol. 4, No. 2, pp. 146-154, April 2006.

[15] M. Sugeno, “ On improvement of stability conditions for continuous Mamdani-like fuzzy systems," IEEE Trans. on System, Man and Cybernetics, part B vol. 34, No. 1, pp. 120-131, 2004.

[16] J.H. Park, Gwi-Tae Park, Seong-Hwan, C. J. Moon, "Direct Adaptive selfstructuring fuzzy controller for nonaffine nonlinear systems, ” Fuzzy Sets System, vol. 153, pp. 429-445, 2005.

[17] S. Laboid. T.H. Guerra, "Adaptive fuzzy control of a class of SISO nonaffine nonlinear systems," Fuzzy Sets System, vol. 158, pp. 1126-1137, 2007.

[18] Shun. F. Su, J. C. Chang and S.S. Chen, "The Study of Direct Adaptive Fuzzy Controllers," Intl Jour. of Fuzzy Systems, Vol. 8, N0.3, pp. 150159, Sept. 2006

[19] L. X. Wang, Adaptive fuzzy System and Control- Design and Stability Analysis, Prentice Hall, Englewood Cliffs, NJ, 1994

[20] E. H. Mamdani , 'Application of fuzzy algorithm for control of simple plant," Proc. IEEE 12 (1221), pp. 1585-1588, 1974 
[21] Xinhghuo Yu and O. Kaynak, "Sliding Mode Control with Soft Computing : A Suvey," IEEE Trans. on Industrial Electronics, vol. 56, no. 9, September 2009

[22] K. D. Young, V. I. Utkin, U. Ozguner, "A control engineer's guide to Sliding mode control," IEEE Trans. Control Sys. Technol. 7 (3), pp. 328-342, 1999.

[23] V. I. Utkin, “ Variable Structure system with Sliding mode," IEEE Trans. Autom. Control 22 (2), pp. 212-222, 1997

[24] M. Zeinoli, L. Notash, "Adaptive sliding modes control with uncertainty estimation for robot manipulator," Mechanism and Machines, Elsevier, vol. 45, pp 80-90, 2010

[25] Tai $\mathrm{Zu} W u$ and Yau T. Juang, "Adaptive fuzzy sliding modes controller of uncertain nonlinear system," Trans. ISA, Elsevier, vol. 47, pp 279-285, 2008

[26] R. G. Berstecher, Rainer Palm and $H$. D. Unbehauen, "An Adaptive Fuzzy Sliding Mode Controller," IEEE Trans. on Industrial Electronics, vol. 48, no. 1, February 2001

[27] N. Essounbouli and Abdul Hamzaoui, "Direct and Indirect Robust Adaptive Fuzzy Controller for a class of Nonlinear Systems," Intl Journ. of Control, Automat. And Systems, vol4, no. 2, pp. 146-154, April 2006.

[28] A. El. Ougli, I. Lagrat and I. Boumhidi, "Direct Adaptive Fuzzy Control of nonlinear Systems," Journ. ACSE-ICGST, vol.8, Issue II, December 2008.

[29] D. Ling Tsay, H. Yuan Chung and C. Jung Lee, "The Adaptive Control of Nonlinear System Using the Sugeno-Type of Fuzzy Logic," IEEE Trans. Fuzzy System. Vol. 7, No. 2, pp 225-229, April. 1999

[30] C. L. Hwang, "A novel Takagi-Sugeno based robust Adaptive fuzzy sliding mode controller," IEEE Trans. Fuzzy System. Vol. 12, No. 5, pp 676-687, Oct. 2004

[31] Y. W. Liang, S. D. Xu, D. C. Liaw and C.C. Chen, "A study of T-S model based SMC scheme with application to robot control," IEEE Trans. Ind. Electronics, vol 55, No. 11, pp 3964-3971, Nov. 2008

[32] M. S. Park, D. Chwa and S. K. Hong,"Antisway tracking control of overhead crane with system uncertainty and actuator nonlinearity using adaptive fuzzy sliding control," IEEE Ind. Electronics Vol. 55, No. 11, pp 3972-3984, Nov. 2008.

[33] T. J. Procyk, E. H. Mamdani, "A linguistic self-organising process controller, "Automatica, Vol. 15, pp. 15-30, 1979

[34] Gustavo Luiz C. M. de Abreu and J. F. Ribeiro, "A self-organizing fuzzy logic controller for active control of flexible structures using piezoelectric actuators," Applied Soft Computing, Elsevier, Vol. 1, pp.271-283, 2002

[35] Chien H. Lee. and Sheng D.Wang, "A self-organizing adaptive fuzzy controller," Fuzzy Sets System, Elsevier, vol. 80, pp. 295-313, 1996.

[36] Phi A. P. and T. J. Gale, "Direct Adaptive control with a self-structuring algorithm," Fuzzy Sets System, Elsevier, vol. 159, pp. 871-899, 2008.

[37]J. C. Wu and T.S. Liu, "A Sliding-Mode Approach to Fuzzy Control Design," IEEE Trans. Control Sys. Technol. Vol. 4, No. 2, March 1997

[38]Feijun Song Smith, S.M., "A comparison of sliding mode fuzzy controller and fuzzy sliding mode controller," Fuzzy Information Processing Society, NAFIPS, pp. $480-484,2000$

[39] Mudi, R. K., Pal, N. R., “A robust selftuning scheme for PI- and PD-type fuzzy 
controllers," IEEE Trans. on Fuzzy Systems, Vol. 7, No. 1, pp. 2-16, 1999

[40] L. K. Wong, H. F. Leung and Peter K. S., "Stability Analysis of Fuzzy Logic Control Systems," Stability Issues in Fuzzy in Fuzzy Control, edited by Javier Aracil Francisco Gordill, Physica-Verlag, Heildelberg 2000.

[41] H. Hellendron and C. Thomas, "Defuzzification in Fuzzy Controllers," Journals of Intelligence and Fuzzy Systems, Vol. 1, 1993, pp. 109-123.

[42] Z. Kovacic, M. Balenovic and $S$. Bogdan, "Sensitivity based self learning fuzzy logic control for a servosystem," IEEE Control System, June 1998.

[43] K. M. Passino and S. Yurkovich, Fuzzy control, Addison-Wesley Longman, Inc. Calif. 1998 